DOI:10.2478/rrlm-2021-0015

\title{
Predictors associated with increased troponin in acute decompensated and chronic heart failure patients
}

\author{
Anamaria Draghici ${ }^{1,2}$, Catalin Adrian Buzea ${ }^{1,3^{*}}$, Caterina Delcea ${ }^{1,3}$, Ancuta \\ Vijan $^{1,3}$, Gheorghe Andrei Dan ${ }^{1,3}$ \\ 1. Internal Medicine, Carol Davila University of Medicine and Pharmacy, Romania \\ 2. Internal Medicine, Colentina University Hospital, Romania \\ 3. Cardiology, Colentina University Hospital, Romania
}

\begin{abstract}
Background: Myocardial injury (INJ) expressed by elevated high-sensitivity troponin (hs-Tn) is common in heart failure (HF), due to cardiovascular and non-cardiac conditions. The mechanisms of INJ in acute decompensated HF (ADHF) versus chronic HF (CHF) are still debated. This study's purpose was to evaluate the determinants of elevated hs-TnT in ADHF and CHF. Methods: We retrospectively analyzed consecutive HF patients with hs-TnT measured on admission, hospitalized in a tertiary-care hospital. Rehospitalizations, acute coronary syndromes, embolisms, infections, autoimmunity and malignancy were excluded. Cut-off point for hs-TnT was 14 ng/L. Results: Our study included $488 \mathrm{HF}$ patients, $56.55 \%$ with ADHF. Mean age was $72.52 \pm 10.09$ years. $53.89 \%$ were females. $67.75 \%$ ADHF and $45.75 \%$ CHF patients had elevated hs-TnT. Median hs-TnT was higher in ADHF versus CHF (21.05[IQR 12.74-33.81] vs 13.20[IQR 7.93-23.25], $p<0.0001)$. In multivariable analysis in ADHF and CHF, $\log 10 N T$-proBNP $(H R=5.30,95 \% C I$ 2.71-10.38, $p<0.001$, respectively $H R=5.49,95 \% C I 1.71-17.57, p=0.004)$ and eGFR (HR=0.72, 95\%CI 0.62-0.85, $p<0.001$, respectively $H R=0.71,95 \% C I 0.55-0.93, p=0.014)$ were independent predictors for increased $h s-T n T$. Independent factors associated with elevated hs-TnT in ADHF were male sex $(H R=2.52,95 \%$ CI 1.31-4.87, $p=0.006)$ and chronic pulmonary obstructive disease (COPD) $(H R=10.57$, $95 \%$ CI 1.26-88.40, $p=0.029)$, while in CHF were age ( $H R=2.68,95 \% C I 1.42-5.07, p=0.002)$ and previous stroke (HR $=5.35,95 \% C I$ 0.98-29.20, $p=0.053)$. Conclusion: HF severity, expressed by NT-proBNP levels, and kidney disease progression, expressed by eGFR, were independent predictors associated with increased hs-TnT in both ADHF and CHF. Specific independent predictors were also indentified in ADHF (male sex, COPD) and CHF (age, history of stroke).
\end{abstract}

Keywords: predictors, high sensitive troponin, acute decompensated heart failure, chronic heart failure Received: 13 ${ }^{\text {th }}$ February 2021; Accepted: $7^{\text {th }}$ March 2021; Published: $17^{\text {th }}$ March 2021

* Corresponding author: Adrian Catalin Buzea, Internal Medicine, Carol Davila University of Medicine and Pharmacy, Romania. E-mail: adrian_c_buzea@yahoo.com 


\section{Introduction}

Biomarkers attract increased research interest, not only for guiding diagnosis and treatment strategies, but also for stratifying prognosis $(1,2)$. Cardiac troponin $(\mathrm{Tn})$ became an important player on this stage (3), especially since the development of high-sensitivity (hs) assays. In 1997 La Vecchia et al reported for the first time the detection of serum Tn I in patients with HF of non-ischemic origin, correlated with higher NYHA class and worse ventricular function and prognosis (4). Furthermore, the use of hs-Tn allowed an increased detection of myocardial injury associated with HF (5).

Elevated levels of hs-Tn can be identified in both acute decompensated HF (ADHF) and chronic HF (CHF), due to partially different mechanisms, with similar prognostic value (6). In ADHF, decreased coronary perfusion, reduced cardiac output, excessive catecholamine secretion, increased oxidative stress, and disrupted calcium metabolism account for the increased hs-Tn levels $(3,7)$. In CHF, the excessive stimulation of renin-angiotensin-aldosterone system and mechanical stress due to concentric remodeling of the left ventricle are the main drivers of elevated hs-Tn, irrespective of the HF etiology (8).

Increased hs-Tn is not linked only to cardiovascular mechanisms but also to extracardiac factors. In the general population, plasma concentrations of Tn are higher with age and also in men compared to women(9-11). Obesity (12) and diabetes mellitus $(13,14)$ are associated with increased levels of hs-Tn, linked to subclinical myocardial damage and chronic systemic inflammation. Chronic kidney disease (11) induces hs-Tn elevation associated with decreased creatinine clearance(15), microvascular renal disease (16) and uremia, which may contribute to ischemia through endothelial dysfunction, inflammation, oxidative stress, coronary artery calcification or hyperhomocysteinemia (17).
In HF patients, hs-Tn elevation was also associated with lower left ventricle ejection fraction (LVEF) (18), ischemic heart disease (19), and left ventricular hypertrophy, probably as a result of inadequate myocardial perfusion (20).

Although clinical factors associated with increased levels of hs-Tn have been analyzed in heart failure, few data comparing the different mechanisms involved in CHF vs ADHF are available. The purpose of this study was to evaluate the association between different clinical and biological parameters and the rise of hs-TnT concentration in ADHF and CHF.

\section{Materials and Methods}

\section{Type of study}

This is an observational, retrospective cohort study. The study protocol, in compliance with the principles of the Declaration of Helsinki, was approved by the Hospital's Ethical Committee on October 10th 2018.

\section{Population}

HF patients admitted consecutively to the Cardiology Department of a tertiary-care hospital from January 2011 to December 2014, included in the HI-HF (Hematological Indices in Heart Failure) cohort were considered for inclusion. Methodology for inclusion in this database, detailed in a previous manuscript awaiting publication, consisted of patients' electronic health records being available, patients' consent on admission for their data to be usable for research purposes, NT-proBNP levels measured on day one of hospital admission and echocardiographic examination documented during the index hospitalization. An additional inclusion criterion for the current study was the measurement of hsTnT on the day of admission.

Exclusion criteria included rehospitalizations and significant comorbidities that are known to modify the cardiac biomarkers: acute coronary 
syndromes, pulmonary embolisms, infections, autoimmune diseases either active or under treatment with systemic corticosteroids, active malignancy, concomitant chemotherapy or radiotherapy and moderate-severe anemia.

Patients were included in this cohort for two purposes. The first, materialized in the current manuscript, was to assess the determinants of myocardial injury translated by increased troponin levels in HF patients. The second aim was to study the impact of myocardial injury on longterm survival prognosis of these patients, which will be assessed in a separate study.

\section{Definitions}

Patients were divided in 2 subgroups: ADHF and $\mathrm{CHF}$, according to the onset of worsening symptoms. Those with declining clinical HF parameters during the previous 1 month before admission were considered ADHF (2). All others were considered CHF.

According to current ESC guidelines, HF was categorized as reduced ejection fraction (HFrEF), mid-range EF (HFmrEF) and preserved EF (HFpEF).

Atrial fibrillation (AF) diagnosis included paroxysmal, persistent, and permanent $\mathrm{AF}$ as well as atrial flutter.

All diagnoses were collected from the medical records as were established by the treating physician at the time of hospital discharge.

\section{Clinical and biological parameters}

Data collected included patients' demographics, medical history, clinical and laboratory parameters, standard echocardiographic measurements and treatment recommendations at discharge. hs-TnT concentrations were determined using the electrochemiluminescence method (Roche Diagnostics Elecsys ${ }^{\circledR}$ Troponin T STAT assay). This assay had a limit of detection of $5 \mathrm{ng} / \mathrm{L}$ and a 99th percentile cutoff point for a healthy reference population of $14 \mathrm{ng} / \mathrm{L}$ (21).
Estimated glomerular filtration rate (eGFR) was calculated using the Chronic Kidney Disease Epidemiology Collaboration (CKD-EPI) formula (22).

\section{Statistical analysis}

NCSS 12 Statistical Software (NCSS, LLC. Kaysville, Utah, USA) and EpiInfo version 7.2.1.0 were used for data analysis. A p-value less than 0.05 was considered statistically significant.

Numerical variables with a Gaussian distribution were expressed as mean \pm standard deviation. Numerical variables with non-Gaussian distribution were expressed as median [interquartile range]. Categorical variables were expressed as absolute numbers and percentages.

The ANOVA test was used for comparison of numerical variables with homogeneous variance and Mann-Whitney/Wilcoxon two-sample test for those with inhomogeneous variance. The Chi-square corrected (Yates) test was used to analyze correlations of dichotomous variables. The Spearman coefficient was used to determine bivariate correlations.

Using the $14 \mathrm{ng} / \mathrm{L}$ cut-off value, hs-TnT was transformed into a dichotomous variable and analyzed accordingly throughout the study.

All parameters with significant association to increased hs-TnT in univariate analysis were included in a multivariable logistical regression. Independent predictors for increased hs-TnT were established using the Forward conditional method. Logarithmic transformation in the base of 10 of NT-proBNP was used in the logistical regression to optimize the analysis of this highly variable parameter with skewed distribution.

\section{Results}

Our study included 488 HF patients, of which $56.55 \%$ had ADHF (Figure 1). Mean age was $72.52 \pm 10.09$ years, with older patients in the 


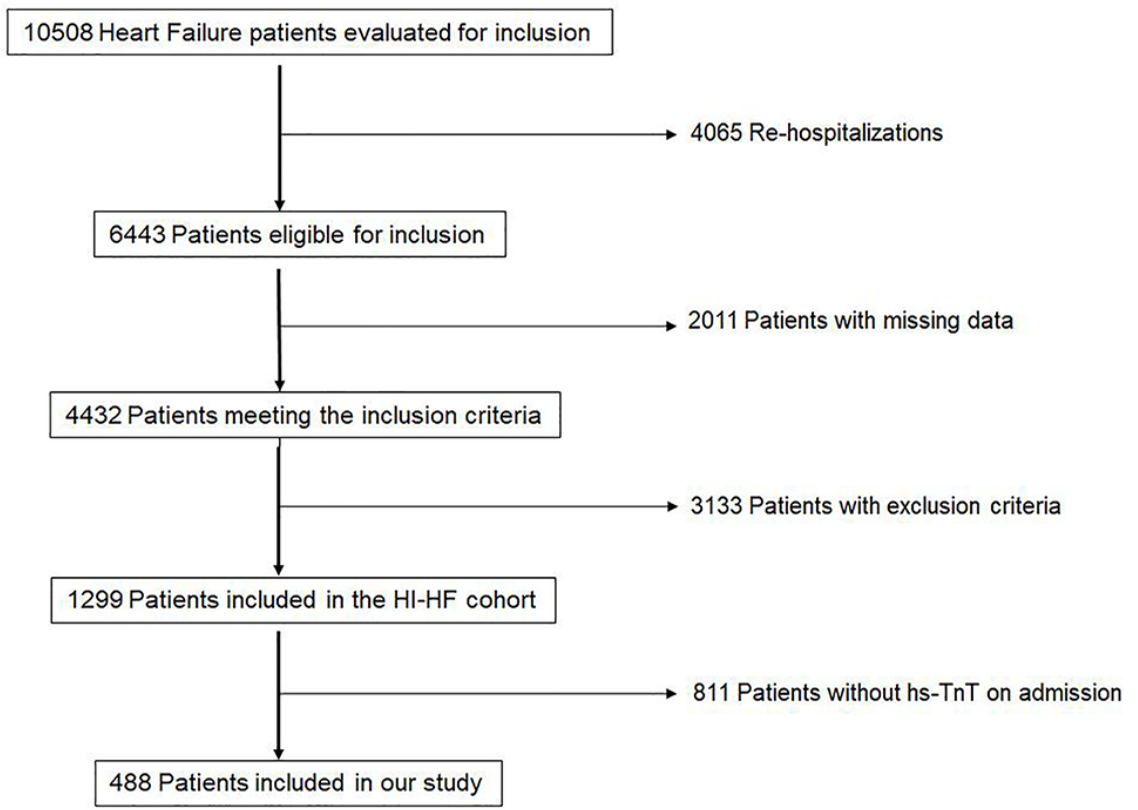

Fig. 1. PRISMA flow chart for patients selection

ADHF group, compared to those with CHF. Female gender was slightly predominant in both $\mathrm{ADHF}$ and CHF groups. The general characteristics are shown in Table 1. Patients presenting with CHF were more likely to have HFpEF, while in the group of ADHF, HFrEF was prevailing (Table 1). Among cardiovascular risk factors and comorbidities arterial hypertension, dyslipidemia, ischemic heart disease, atrial fibrillation and chronic kidney disease were highly frequent (Table 1).

Of the 488 patients in our cohort, elevated levels of hs-TnT were observed in 58.2\% patients. The mean hs-TnT concentration was significantly higher in ADHF population compared to the CHF cohort.

In ADHF patients the univariate analysis showed an association between elevated hs-TnT and older age, male sex, clinical signs of congestion, dyspnea at rest, chronic kidney disease, moderate to severe mitral regurgitation and COPD (Table 2). The subgroup with high hs-TnT had significantly lower eGFR and sodium levels, higher
NT-proBNP levels, lower LVEF, increased LV diameters, increased right heart chamber diameters and higher systolic pulmonary arterial pressures (SPAP) (Table 3). The prevalence of dyslipidemia was lower in the high hs-TnT subgroup. Bivariate correlations were found between hs-TnT levels and NT-proBNP and eGRF (Supplemental Table 1). In multivariable regression analysis male sex, NT-proBNP, eGFR and chronic pulmonary obstructive disease were independent predictors for increased hs-TnT in the ADHF group (Table 4).

In CHF patients, we observed an association between elevated hs-TnT and older age, male sex, clinical congestion, dyspnea at rest, chronic kidney disease, moderate to severe mitral regurgitation or severe aortic stenosis and stroke. In the high hs-TnT subgroup some cardiovascular risk factors (arterial hypertension, dyslipidemia, obesity) and stable ischemic heart disease were less prevalent compared to the normal troponin subgroup (Table 2). Higher hs-TnT was associated with decreased eGRF, lower hemoglobin, 
Table 1. General characteristics

\begin{tabular}{|c|c|c|c|c|}
\hline & All patients $n=488$ & ADHF $n=276$ & CHF $n=212$ & p value \\
\hline Age [years] & $72.52 \pm 10.09$ & $73 \pm 9.71$ & $71.03 \pm 10.40$ & $<0.001$ \\
\hline Sex $[\%$ female $]$ & $53.89 \%(263)$ & $55.43 \%(153)$ & $51.89 \%(110)$ & 0.43 \\
\hline Length of hospital stay [days]* & $6.63 \pm 4.42$ & $7.44 \pm 5.14$ & $5.63 \pm 3.12$ & $<0.001$ \\
\hline In-hospital mortality [\%] & $3.69 \%(18)$ & $6.52 \%(18)$ & $0 \%(0)$ & $<0.001$ \\
\hline \multicolumn{5}{|l|}{ Heart failure characteristics } \\
\hline $\operatorname{HFrEF}[\%]$ & $35.14 \%(156)$ & $43.89 \%(115)$ & $22.53 \%(41)$ & $<0.001$ \\
\hline HFmrEF [\%] & $31.98 \%(142)$ & $33.59 \%(88)$ & $29.67 \%(54)$ & 0.38 \\
\hline HFpEF $[\%]$ & $32.88 \%(146)$ & $22.52 \%(59)$ & $47.80 \%(87)$ & $<0.001$ \\
\hline NYHA III [\%] & $50.12 \%(212)$ & $62.65 \%(161)$ & $30.72 \%(51)$ & $<0.001$ \\
\hline NYHA IV [\%] & $17.21 \%(84)$ & $29.35 \%(81)$ & $1.42 \%(3)$ & $<0.001$ \\
\hline \multicolumn{5}{|l|}{ Cardiovascular risk factors } \\
\hline Arterial hypertension [\%] & $79.10 \%(386)$ & $80.80 \%(223)$ & $76.89 \%(163)$ & 0.29 \\
\hline Dyslipidemia [\%] & $63.31 \%(309)$ & $59.42 \%(164)$ & $68.39 \%(145)$ & 0.04 \\
\hline Obesity $[\%]$ & $41.39 \%(202)$ & $44.20 \%(122)$ & $37.74 \%(80)$ & 0.15 \\
\hline Diabetes mellitus [\%] & $30.74 \%(150)$ & $32.61 \%(90)$ & $28.3 \%(60)$ & 0.30 \\
\hline \multicolumn{5}{|l|}{ Medical history } \\
\hline Ischemic heart disease $[\%]$ & $44.26 \%(216)$ & $43.84 \%(121)$ & $44.81 \%(95)$ & 0.83 \\
\hline Prior MI $[\%]$ & $25.82 \%(126)$ & $28.62 \%(79)$ & $22.17 \%(47)$ & 0.10 \\
\hline Stable angina $[\%]$ & $20.70 \%(101)$ & $19.93 \%(55)$ & $21.70 \%(46)$ & 0.63 \\
\hline History of stroke or TIA [\%] & $11.89 \%(58)$ & $13.04 \%(36)$ & $10.38 \%(22)$ & 0.36 \\
\hline Atrial fibrillation $[\%]$ & $47.34 \%(231)$ & $57.97 \%(160)$ & $33.49 \%(71)$ & $<0.001$ \\
\hline Chronic kidney disease [\%] & $58.81 \%(287)$ & $62.68 \%(173)$ & $53.77 \%(114)$ & 0.04 \\
\hline $\begin{array}{l}\text { Moderate to severe mitral regur- } \\
\text { gitation }[\%]\end{array}$ & $16.33 \%(65)$ & $24.05 \%(57)$ & $4.97 \%(8)$ & $<0.001$ \\
\hline Severe aortic stenosis [\%] & $3.18 \%(11)$ & $5.11 \%(10)$ & $0.72 \%(1)$ & 0.93 \\
\hline COPD [\%] & $6.35 \%(31)$ & $7.97 \%(22)$ & $4.25 \%(9)$ & 0.09 \\
\hline \multicolumn{5}{|l|}{ Laboratory findings } \\
\hline Haemoglobin $[\mathrm{g} / \mathrm{dL}]$ & 13.10 [IQR 11.82-14.50] & 12.80 [IQR 11.52-14.20] & 13.50 [IQR 12.10-14.60] & 0.001 \\
\hline Creatinine $[\mathrm{mg} / \mathrm{dL}]$ & $0.94[$ IQR $0.80-1.20]$ & 0.97 [IQR 0.80-1.30] & $0.91[$ IQR $0.79-1.10]$ & 0.31 \\
\hline $\mathrm{GFR}\left[\mathrm{ml} / \mathrm{min} / 1.73 \mathrm{~m}^{2}\right]$ & 68.11 [IQR 49.97-87.37] & 65.52 [IQR 47.45-84.59] & 74.89 [IQR 56.06-189.39] & $<0.001$ \\
\hline Serum sodium $[\mathrm{mmol} / \mathrm{L}]$ & 140 [IQR 138-143] & 140 [IQR 138-143] & 140 [IQR 138-142] & 0.77 \\
\hline Serum potassium $[\mathrm{mmol} / \mathrm{L}]$ & 4.37 [IQR 4.04-4.68] & 4.38 [IQR 4.05-4.72] & 4.36 [IQR 4.03-4.63] & 0.17 \\
\hline NT-proBNP [pg/mL] & $\begin{array}{c}1499.00 \\
{[\mathrm{IQR} \text { 602.50-4358.50] }}\end{array}$ & $\begin{array}{c}2924.00 \\
{[\text { IQR 956.40-6951.00] }}\end{array}$ & $\begin{array}{c}889.80 \\
\text { [IQR 359.30-1785.00] }\end{array}$ & $<0.001$ \\
\hline hs-cTnT [ng/mL] & 17.11 [IQR 10.30-29.24] & 21.05 [IQR 12.74-33.81] & 13.20 [IQR 7.93-23.25] & $<0.001$ \\
\hline Elevated hsTnT [\%] & $58.20 \%(284)$ & $67.75 \%(187)$ & $45.75 \%(97)$ & $<0.001$ \\
\hline \multicolumn{5}{|l|}{ Echocardiographic parameters } \\
\hline $\operatorname{LVEF}[\%]$ & $41.49 \pm 12.33$ & $38.52 \pm 12.48$ & $45.78 \pm 10.78$ & $<0.001$ \\
\hline $\mathrm{LAD}[\mathrm{mm}]$ & $44.17 \pm 7.4$ & $46.08 \pm 7.64$ & $41.45 \pm 6.10$ & $<0.001$ \\
\hline LVDD $[\mathrm{mm}]$ & $52.66 \pm 8.12$ & $53.60 \pm 8.65$ & $51.39 \pm 7.11$ & $<0.01$ \\
\hline LVSD [mm] & $34.42 \pm 9.04$ & $39.69 \pm 9.39$ & $36.61 \pm 8.20$ & $<0.001$ \\
\hline IVS $[\mathrm{mm}]$ & $12.32 \pm 2.14$ & $12.38 \pm 2.06$ & $12.23 \pm 2.24$ & 0.50 \\
\hline Diastolic dysfunction & $79.74 \%(185)$ & $73.68 \%(84)$ & $85.59 \%(101)$ & 0.41 \\
\hline Grade II $[\%]$ & $7.84 \%(16)$ & $8.91 \%(9)$ & $6.80 \%(7)$ & 0.57 \\
\hline Grade III [\%] & $10.29 \%(21)$ & $15.84 \%(16)$ & $4.85 \%(5)$ & $<0.01$ \\
\hline \multicolumn{5}{|l|}{ Wall motion abnormalities } \\
\hline Segmental abnormalities [\%] & $30.70 \%(109)$ & $34.67 \%(69)$ & $25.64 \%(40)$ & 0.07 \\
\hline Diffuse abnormalities [\%] & $47.61 \%(169)$ & $49.25 \%(98)$ & $45.51 \%(71)$ & 0.49 \\
\hline Pericardial effusion $[\%]$ & $12.38 \%(39)$ & $17.51 \%(31)$ & $5.08 \%(8)$ & 0.25 \\
\hline $\mathrm{RAD}[\mathrm{mm}]$ & $40.23 \pm 7.13$ & $42.12 \pm 7.48$ & $37.53 \pm 5.63$ & $<0.001$ \\
\hline $\operatorname{RVD}[\mathrm{mm}]$ & $32.89 \pm 5.87$ & $33.98 \pm 5.88$ & $31.34 \pm 5.50$ & $<0.001$ \\
\hline $\mathrm{SPAP}[\mathrm{mmHg}]$ & $40.51 \pm 13.92$ & $44.08 \pm 14.64$ & $33.91 \pm 9.50$ & $<0.001$ \\
\hline
\end{tabular}

ADHF-acute decompensated heart failure; CHF-chronic heart failure; HFrEF-heart failure with reduced ejection fraction; HFmEF-heart failure with mid-range ejection fraction; HFpEF-heart failure with preserved ejection fraction; eGFR-estimated glomerular filtration rate; NT-proBNP-N-terminal pro b-type natriuretic peptide; hs-TnT-high sensitive troponin T, LVEF-left ventricle ejection fraction, LAD-left atrium diameter, LVDD-left ventricle diastolic diameter, LVSD-left ventricle systolic diameter, IVS-interventricular septum, RAD-right atrium diameter, RVD-right ventricle diameter, SPAP-systolic pulmonary artery pressure. The number in the round brackets represent the number of patients *For patients surviving hospitalization 
Table 2. Clinical determinants of elevated hsTnT

\begin{tabular}{lcccccc}
\hline & $\begin{array}{c}\text { Normal } \\
\text { hsTnT } \\
\text { N= 89 }\end{array}$ & $\begin{array}{c}\text { ADHF } \\
\text { Elevated } \\
\text { hsTnT } \\
\mathbf{N = 1 8 7}\end{array}$ & p value & $\begin{array}{c}\text { Normal } \\
\text { hsTnT } \\
\mathbf{N = 1 1 5}\end{array}$ & $\begin{array}{c}\text { CHF } \\
\text { Elevated } \\
\text { hsTnT } \\
\mathbf{N}=\mathbf{9 7}\end{array}$ & $\begin{array}{c}\text { p } \\
\text { value }\end{array}$ \\
& & & & & & \\
\hline Demographics & $71.98 \pm 7.62$ & $74.47 \pm 10.49$ & 0.04 & $67.54 \pm 10.17$ & $75.17 \pm 9.10$ & $<0.001$ \\
\hline Age, years & 65.17 & 50.80 & 0.04 & 60.00 & 42.27 & 0.01 \\
Women (\%) & & & & & \\
\hline Clinical characteristics on admission & 14.61 & 36.36 & $<0.001$ & 0 & 3.09 & 0.05 \\
Dyspnea at rest (\%) & 47.19 & 61.54 & 0.01 & 19.32 & 42.65 & 0.001 \\
Congestion signs (\%) & & & & & & \\
Cardiovascular risk factors & 84.27 & 79.14 & 0.31 & 82.61 & 70.10 & 0.06 \\
HTN (\%) & 60.67 & 47.06 & 0.03 & 80.87 & 58.76 & $<0.001$ \\
Dyslipidemia (\%) & 50.56 & 41.18 & 0.14 & 45.22 & 28.87 & 0.01 \\
Obesity (\%) & & & & & & \\
\hline Medical history & 40.45 & 45.45 & 0.43 & 53.04 & 35.05 & $<0.01$ \\
IHD (\%) & 23.60 & 31.02 & 0.20 & 23.48 & 20.62 & 0.61 \\
$\quad$ Prior MI (\%) & 16.85 & 21.39 & 0.37 & 28.70 & 13.40 & $<0.01$ \\
$\quad$ SA (\%) & 11.24 & 13.90 & 0.53 & 5.22 & 16.49 & $<0.01$ \\
Prior stroke/ TIA (\%) & 46.07 & 70.59 & $<0.001$ & 36.52 & 74.23 & $<0.001$ \\
CKD (\%) & 14.63 & 29.03 & 0.01 & 3.19 & 7.46 & 0.04 \\
Moderate/severe MR (\%) & 3.17 & 7.07 & 0.13 & 0 & 1.89 & $<0.01$ \\
Severe AS (\%) & 2.25 & 10.70 & 0.01 & 5.22 & 3.09 & 0.44 \\
COPD (\%) &
\end{tabular}

ADHF - acute decompensated heart failure, CHF - chronic heart failure, HTN - hypertension, IHD - ischemic heart disease, MI - myocardial infarction, SA - stable angina, TIA - transitory ischemic attack, CKD - chronic kidney disease, MR - mitral regurgitation, AS - aortic stenosis, COPD - chronic obstructive pulmonary disease

higher glycemic levels, higher NT-proBNP levels, higher SPAP, greater LV wall thickness, diffuse LV wall motion abnormalities, restrictive diastolic dysfunction and pericardial effusion (Table 3). Bivariate correlations were found between hs-TnT levels and age, NT-proBNP and eGRF (Supplemental Table 1). In the CHF group multivariable analysis identified age, NT-proB$\mathrm{NP}$, eGFR and previous stroke as independent predictors for increased hs-TnT (Table 4).

\section{Discussion}

We aimed to identify and compare the factors associated with increased hs-TnT levels in two different clinical settings, namely ADHF and CHF. The main finding of this study was the strong relation of elevated levels of hs-TnT to NT-proB-
NP and renal function in both clinical scenarios, but also with specific predictors for each setting. Our study highlights that elevated levels of hsTnT, a marker for myocardial injury, can be a common feature for HF patients, with significantly higher values in ADHF. Similar results have been described previously, detectable hsTnT being present in patients with HF, irrespective of the acute or chronic scenario, with higher levels in ADHF (23-25). In a comprehensive review, Kociol et al identified an incidence of detectable, circulating troponin levels in ADHF patients ranging from $6.2 \%$ up to $83.9 \%$, linked to prognosis (3). In a sub-analysis of the ASCEND-HF study, detectable levels of hs-TnI were observed in more than $2 / 3$ of the studied population with elevated concentrations in $50 \%$ of the cases (24). Increased hs-TnT concentra- 


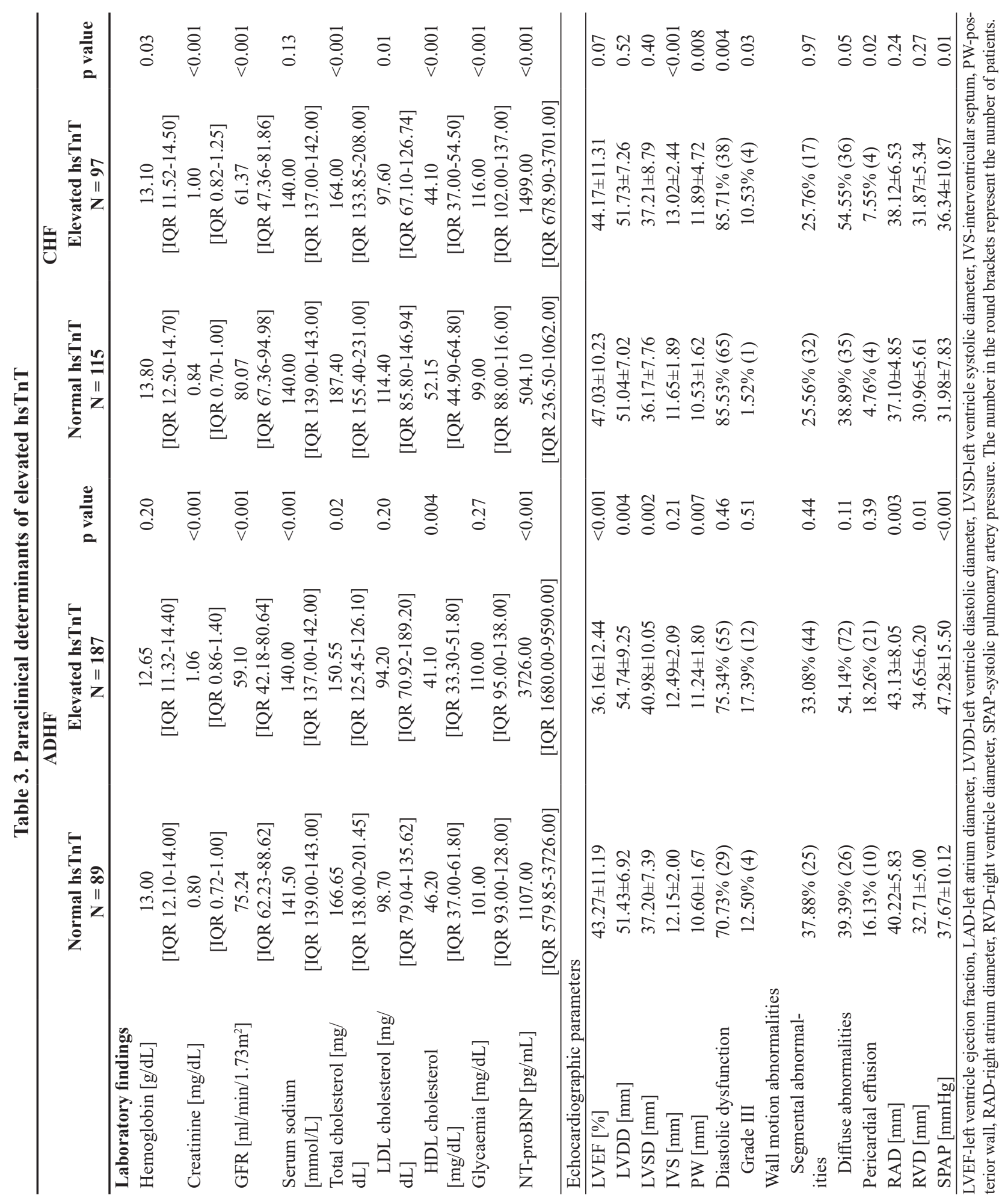


Table 4. Multivariable logistic regression for $\mathrm{ADHF}$ and CHF groups

\begin{tabular}{llccc}
\hline & HR & $\mathbf{9 5 \%} \mathbf{C I}$ & p value \\
\hline ADHF & & & $<0.001$ \\
& LogNT-proBNP & 5.30 & $2.71-10.38$ & $<0.001$ \\
& eGFR & 0.72 & $0.62-0.85$ & 0.006 \\
& Sex & 2.52 & $1.31-4.87$ & 0.029 \\
& COPD & 10.57 & $1.26-88.40$ & 0.004 \\
\hline CHF & & & 0.014 \\
& LogNT-proBNP & 5.49 & $1.71-17.57$ & 0.002 \\
& eGFR & 0.71 & $0.55-0.93$ & 0.053 \\
& Age & 2.68 & $1.42-5.07$ & \\
& Previous stroke & 5.35 & $0.98-29.20$ &
\end{tabular}

COPD - Chronic Obstructive Pulmonary Disease, NT-proBNP - N-terminal prohormone of brain natriuretic peptide eGFR - estimated glomerular filtration rate. Factors also included in the regression without independent significance: age, signs of congestion, NYHA IV, moderate to severe mitral regurgitation, left ventricle ejection fraction, posterior wall of left ventricle, left ventricle diastolic diameter, pulmonary hypertension

tion is a prevalent finding in ADHF patients, across the LVEF spectrum, with higher levels more frequently identified in patients with reduced EF $(7,19)$.

Myocardial injury is therefore common in HF, with important prognostic value (2). There are many hypotheses regarding the release of $\mathrm{Tn}$ in $\mathrm{ADHF}$, the most relevant taking in consideration reduced cardiac output and increased ventricular pressures or ventricular remodeling and neurohormonal stimulation (26). The result is subclinical ischemia, which in turn induces hs-TnT release from affected myocytes, possibly even before necrosis, and more so afterwards (27). Myocardial strain, and chronic ischemia, both due to insufficient supply as well as increased demand, also contribute to elevation of troponin levels in CHF (28).

Due to documented variability, there are proposals for age and sex specific cut-offs for Tn assay, with a higher value for advanced age and male gender $(9,10)$. This differentiation has contributed to a gender uniformization in the diagnosis and management of myocardial injury (29). Older age and male sex were also correlated to elevated hs-TnT levels in both CHF and ADHF in univariate analysis in our study. In multi- variable analysis, male gender remained an independent predictor for hs-TnT concentration only in ADHF, while advanced age only in CHF. ADHF patients were overall significantly older than CHF ones, which could explain the loss of independent prediction power due to generally increased ages in this subgroup. The relation of age and hs-TnT levels was also stronger in bivariate correlation in $\mathrm{CHF}$ compared to $\mathrm{ADHF}$. In both groups, hs-TnT concentrations were correlated to more severe forms of disease and worse functional status. In univariate analysis, increased hs-TnT was correlated with dyspnea at rest, clinical signs of congestion and increased NT-proBNP levels. After multiple logistic regression, the only indicator independently predictive for elevated hs-TnT in both acute and chronic HF was the NT-proBNP concentration. Furthermore, the logarithmic transformation of NT-proBNP was the strongest independent common predictor of elevated hs-TnT in both subgroups and hs-TnT levels were directly correlated to NT-proBNP levels. Since the hemodynamic overload in HF leads to both increase in circulating NT-proBNP as well as hs-TnT levels (30), their interdependence is expected due to common pathophysiological mechanisms 
leading to their release. Moreover, NT-proBNP levels account for the entire HF clinical and paraclinical spectrum, directly dependent on its severity (31), therefore also extended to covering other signs or symptoms.

The second strongest independent predictor of elevated hs-TnT in both ADHF and CHF patients in our study was eGFR. The relation between renal function and hs-Tn is well known, more so when patients associate HF, warranting caution in integrating the values in this context (32). The underlying mechanisms are still incompletely characterized, however multiple hypotheses concur to explain this association. Low-grade inflammation, myocyte necrosis, cellular turnover, raised cellular permeability, and protein fragmentation all interact in $\mathrm{HF}$ and $\mathrm{CKD}$, leading to increased troponin levels (32-34). Myocardial injury integrated in the cardio-renal syndrome and decreased kidney clearance most probably partake in this complex process (32). The novelty of our study is proving the independent correlation of eGFR to elevated hs-TnT, both in $\mathrm{ADHF}$ and $\mathrm{CHF}$, strengthening the importance of kidney disease in the cardiac homeostasis.

Our study also revealed two more independent predictors of elevated hs-TnT, respectively COPD in ADHF and history of stroke in CHF. In stable COPD patients, increased hs-TnT levels were previously correlated to systemic inflammation, older age, and pulmonary artery pressure (35). In acute COPD, apart from severe infection, elevated hs-TnT was also attributed to type II myocardial injury secondary to hypoxia, tachycardia, or acute right ventricular dysfunction (36). Studies of COPD patients revealed worst outcomes in patients with increased troponin levels (36), and now our study is showing the reversed relation, with COPD increasing the risk profile of HF patients. Since most COPD patients in our sample presented with ADHF, we consider this to be the reason why the indepen- dent predictive value for increased hs-TnT was detected only in the ADHF subgroup.

Chronic myocardial injury expressed by increased troponin levels was precedently correlated to comorbidity burden, including a history of stroke, and it predicted the risk of developing stroke in a large cohort of patients presenting with chest pain (37). Increased hs-TnT levels are also correlated to the severity and extension of atherosclerotic plaques (38). We therefor hypothesize that a history of stroke in the studied CHF patients is associated to a higher atherosclerotic burden and vascular risk, leading to elevated hs-TnT concentrations.

\section{Limitations}

Our study has several limitations, the first being the retrospective design of the study, as well as the timeframe for patient inclusion, justified by the intention to further analyze the long-term survival of these patients in a future study.

Another important mention is that for all patients we analyzed only the first value of the hs-TnT determined on admission.

Our analysis included the LVEF as a numerical, continuous variable. We did not split the patient population into the three categories of reduced, mid-range, and preserved EF, to prevent excessive subgrouping.

\section{Conclusions}

The clinical utility of hs-TnT in defining myocardial injury in HF should be integrated in each patient's individualized clinical context. While many clinical and biological parameters are linked to increased hs-TnT levels, only some of them play a significant and independent role.

HF severity, expressed by NT-proBNP levels, and kidney disease progression, expressed by eGFR, were the strongest independent drivers of increased hs-TnT in our study, in all patients. Elevated hs-TnT levels were also independently 
correlated to male sex and COPD in ADHF and to age and history of stroke in CHF.

\section{Abbreviations}

ADHF- acute decompensated heart failure;

AS- aortic stenosis;

ASCEND-HF- Acute Study of Clinical Effectiveness of Nesiritide in Decompensated Heart

Failure;

CHF- chronic heart failure;

CKD- chronic kidney disease;

CKD-EPI- Chronic Kidney Disease Epidemiology Collaboration;

COPD- chronic pulmonary obstructive disease;

eGFR- estimated glomerular filtration;

HI-HF cohort- Hematological Indices in Heart

Failure cohort;

HF- heart failure;

HFmrEF- heart failure with mid-range ejection fraction;

HFpEF- heart failure with preserved ejection fraction;

HFrEF- heart failure with reduced ejection fraction; hs-Tn- high-sensitivity troponin;

HTN- hypertension;

IHD- ischemic heart disease;

INJ- Myocardial injury;

IVS- interventricular septum;

LAD- left atrium diameter;

LVEF- left ventricle ejection fraction;

LVDD- left ventricle diastolic diameter;

LVSD- left ventricle systolic diameter;

MI- myocardial infarction;

MR- mitral regurgitation;

NT-proBNP- N-terminal pro b-type natriuretic peptide;

NYHA- New York Heart Association;

PW- posterior wall;

RAD- right atrium diameter;

RVD- right ventricle diameter;

SA- stable angina;

SPAP- systolic pulmonary artery pressure;

TIA- transitory ischemic attack

\section{Acknowledgements}

The authors are thankful to Daha I, MD, PhD, Andrus A, MD, Breha A, MD, Hogea I, MD, Niculescu L, MD, Lupan M, MD, Lefter A, MD, Pavalean M, MD, Moise A, MD, and Angheluta $\mathrm{S}, \mathrm{MD}$, for their help in data collection and database completion.

\section{Authors' Contribution}

AD: protocol design, data collection, data analysis and interpretation, manuscript drafting and revision

CAB: protocol design, data analysis and interpretation, manuscript drafting and revision

CD: protocol design, data collection, data analysis and interpretation, manuscript drafting and revision

AV: data collection, data analysis and interpretation, manuscript drafting and revision

GAD: protocol design, data interpretation, manuscript drafting and revision, $\mathrm{PhD}$ supervisor

\section{Conflict of Interest}

All the authors declare no conflict of interest.

\section{References}

1. Yancy CW, Jessup M, Bozkurt B, Butler J, Casey DE, Colvin MM, et al. 2017 ACC/AHA/HFSA Focused Update of the 2013 ACCF/AHA Guideline for the Management of Heart Failure: A Report of the American College of Cardiology/American Heart Association Task Force on Clinical Practice Guidelines and the Heart Failure Society of Amer. J Am Coll Cardiol. 2017;70(6):776-803. DOI: 10.1016/j.jacc.2017.04.025

2. Ponikowski P, Voors AA, Anker SD, Bueno H, Cleland JGF, Coats AJS, et al. 2016 ESC Guidelines for the diagnosis and treatment of acute and chronic heart failure. Eur Heart J. 2016 Jul;37(27):2129-200. DOI: 10.1093/eurheartj/ehw128

3. Kociol RD, Pang PS, Gheorghiade M, Fonarow GC, O'Connor C, Felker GM, et al. Troponin Elevation in Heart Failure- Prevalence, Mechanisms, and Clinical 
Implications. J Am Coll Cardiol. 2010;56(14):1071-8. DOI: 10.1016/j.jacc.2010.06.016

4. La Vecchia L, Mezzena G, Ometto R, Finocchi G, Bedogni F, Soffiati G, et al. Detectable serum troponin I in patients with heart failure of nonmyocardial ischemic origin. Am J Cardiol. 1997;80(1):88-90. DOI: 10.1016/ S0002-9149(97)00291-9

5. Missov E, De Marco T. Clinical insights on the use of highly sensitive cardiac troponin assays. Clin Chim Acta. 1999 Jun;284(2):175-85. DOI: 10.1016/S00098981(99)00079-0

6. Januzzi JL, Filippatos G, Nieminen M, Gheorghiade M. Troponin elevation in patients with heart failure: On behalf of the third Universal Definition of Myocardial Infarction Global Task Force: Heart Failure Section. Vol. 33, European Heart Journal. 2012. p. 2265-71. DOI: 10.1093/eurheartj/ehs191

7. Diez M, Talavera ML, Conde DG, Campos R, Acosta A, Trivi MS, et al. High-sensitivity troponin is associated with high risk clinical profile and outcome in acute heart failure. Cardiol J. 2016;23(1):78-83. DOI: 10.5603/CJ.a2015.0058

8. Shah KS, Maisel AS, Fonarow GC. Troponin in Heart Failure. Heart Fail Clin. 2018;14(1):57-64. DOI: 10.1016/j.hfc.2017.08.007

9. Carlsson AC, Bandstein N, Roos A, Hammarsten O, Holzmann MJ. High-sensitivity cardiac troponin $\mathrm{T}$ levels in the emergency department in patients with chest pain but no myocardial infarction. Int $\mathrm{J}$ Cardiol. 2017;228:253-9. DOI: 10.1016/j.ijcard.2016.11.087

10. Gore MO, Seliger SL, Christopher R, Nambi V, Christenson RH, Hashim IA, et al. Age- and Sex-Dependent Upper Reference Limits for the High-Sensitivity Cardiac Troponin T Assay. 2014;63(14):1441-8. DOI: 10.1016/j.jacc.2013.12.032

11. Bargnoux A, Kuster N, Moréna M, Baptista G, Chenine $\mathrm{L}$, Badiou S, et al. L ' âge et la fonction rénale sont-ils des limites à 1 ' interprétation des biomarqueurs cardiaques ? Ann Biol Clin. 2016;74(4):413-9. DOI: 10.1684/ abc.2016.1165

12. Ndumele C, Cobb L, Lazo M, Bello N, Shah A, Nambi V, et al. Weight History and Subclinical Myocardial Damage. Clin Chem. 2019;64(1):201-9. DOI: 10.1373/ clinchem.2017.282798

13. Zheng J, Ye P, Luo L, Xiao W, Xu R, Wu H, et al. Association between blood glucose levels and high-sensitivity cardiac troponin $\mathrm{T}$ in an overt cardiovascular disease-free community-based study. Diabetes Res Clin Pract. 2012;97(1):139-45. DOI: 10.1016/j.diabres.2012.04.021

14. Hijazi Z, Siegbahn A, Andersson U, Lindahl B, Granger CB, Alexander JH, et al. Comparison of Cardiac Troponins I and T Measured with High-Sensitivity Methods for Evaluation of Prognosis in Atrial Fibrillation : An ARISTOTLE Substudy. 2015;61:368-78. DOI: 10.1373/clinchem.2014.226936

15. Chen M, Gerson H, Eintracht S, Nessim SJ, Macnamara E. Effect of Hemodialysis on Levels of High-Sensitivity Cardiac Troponin T. Am J Cardiol. 2017;120(11):20614. DOI: 10.1016/j.amjcard.2017.08.026

16. Kirkman DL, Muth BJ, Ramick MG, Townsend RR, Edwards DG. Role of mitochondria-derived reactive oxygen species in microvascular dysfunction in chronic kidney disease. Am J Physiol Ren Physiol. 2018;314:423-9. DOI: 10.1152/ajprenal.00321.2017

17. Cai Q, Mukku VK, Ahmad M. Coronary Artery Disease in Patients with Chronic Kidney Disease : A Clinical Update. 2013;(Mi):331-9. DOI: 10.2174/1573403X10 666140214122234

18. Santhanakrishnan R, Chong JPC, Ng TP, Ling LH, Sim $\mathrm{D}$, Leong KTG, et al. Growth differentiation factor 15 , ST2, high-sensitivity troponin $\mathrm{T}$, and N-terminal pro brain natriuretic peptide in heart failure with preserved vs . reduced ejection fraction. Eur J Heart Fail. 2012;14:1338-47. DOI: 10.1093/eurjhf/hfs130

19. Pandey A, Golwala H, Sheng S, DeVore AD, Hernandez AF, Bhatt DL, et al. Factors associated with and prognostic implications of cardiac troponin elevation in decompensated heart failure with preserved ejection fraction: Findings from the American Heart Association Get With the Guidelines-Heart Failure program. JAMA Cardiol. 2017;2(2):136-45. DOI: 10.1001/jamacardio.2016.4726

20. Löwbeer C, Gustafsson S, Seeberger A, Bouvier F, Hulting J. Serum cardiac troponin $\mathrm{T}$ in patients hospitalized with heart failure is associated with left ventricular hypertrophy and systolic dysfunction. Scand J Clin Lab Invest. 2004;64:667-76. DOI: 10.1080/00365510410003002

21. Jarolim P. High sensitivity cardiac troponin assays in the clinical laboratories. 2015;53(5):635-52. DOI: 10.1515/cclm-2014-0565

22. Levey AS, Stevens LA, Schmid CH, Zhang Y, Castro AF, Feldman HI, et al. A new equation to esti- 
mate glomerular filtration rate. Ann Intern Med. 2009 May;150(9):604-12. DOI: 10.7326/0003-4819-150-9200905050-00006

23. Pascual-Figal D, Casas T, Ordonez-Llanos J, Manzano-Fernández S, Bonaque J, Boronat $\mathrm{M}$, et al. Highly sensitive troponin $\mathrm{T}$ for risk stratification of acutely destabilized heart failure. Am Heart J. 2012;163(6):100210. DOI: 10.1016/j.ahj.2012.03.015

24. Felker GM, Hasselblad V, Tang WHW, Hernandez AF, Armstrong PW, Fonarow GC, et al. Troponin $\mathrm{I}$ in acute decompensated heart failure: insights from the ASCEND-HF study. Eur J Heart Fail. 2012 Nov;14(11):1257-64. DOI: 10.1093/eurjhf/hfs 110

25. Rustamova Y, Dobreanu D. Biomarkers in heart failure: From the bedside back to biology. Rev Rom Med Lab. 2018 Jul;26(3):267-70. DOI: 10.2478/rrlm-2018-0031

26. Harrison N, Favot M, Levy P. The Role of Troponin for Acute Heart Failure. Curr Heart Fail Rep. 2019;16(1):21-31. DOI: 10.1007/s11897-019-0420-5

27. Hickman PE, Potter JM, Aroney C, Koerbin G, Southcott E, Wu AHB, et al. Cardiac troponin may be released by ischemia alone, without necrosis. Vol. 411, Clinica Chimica Acta. Elsevier; 2010. p. 318-23. DOI: 10.1016/j.cca.2009.12.009

28. Park KC, Gaze DC, Collinson PO, Marber MS. Cardiac troponins: From myocardial infarction to chronic disease. Cardiovasc Res. 2017;113(14):1708-18. DOI: $10.1093 / \mathrm{cvr} / \mathrm{cv} x 183$

29. Lee K, Ferry A, Anand A, Strachan F, Chapman A, Kimenai D, et al. Sex-Specific Thresholds of High-Sensitivity Troponin in Patients With Suspected Acute Coronary Syndrome. J Am Coll Cardiol. 2019;74(16):203243. DOI: $10.1016 /$ j.jacc.2019.07.082

30. Fu S, Ping P, Zhu Q, Ye P, Luo L. Brain natriuretic peptide and its biochemical, analytical, and clinical issues in heart failure: A narrative review. Vol. 9, Frontiers in Physiology. Frontiers Media S.A.; 2018. p. 692. DOI: 10.3389/fphys.2018.00692
31. Bettencourt P. NT-proBNP and BNP: biomarkers for heart failure management. Eur J Heart Fail. 2004 Mar;6(3):359-63. DOI: 10.1016/j.ejheart.2004.01.008

32. Long B, Belcher CN, Koyfman A, Bronner JM. Interpreting Troponin in Renal Disease: A Narrative Review for Emergency Clinicians. Am J Emerg Med. 2020;38(5):990-7. DOI: 10.1016/j.ajem.2019.11.041

33. Jeremias A, Gibson CM. Narrative review: Alternative causes for elevated cardiac troponin levels when acute coronary syndromes are excluded. Vol. 142, Annals of Internal Medicine. American College of Physicians; 2005. p. 786-91. DOI: 10.7326/0003-4819-142-9200505030-00015

34. Kanderian AS, Francis GS. Cardiac troponins and chronic kidney disease. Vol. 69, Kidney International. Kidney Int; 2006. p. 1112-4. DOI: 10.1038/sj. ki. 5000174

35. Kida K, Hattori K, Ishii T, Motegi T, Kusunoki Y, Gemma A, et al. Relationship between serum cardiac troponin $\mathrm{T}$ level and cardiopulmonary function in stable chronic obstructive pulmonary disease. Int J Chron Obstruct Pulmon Dis. 2015 Feb;10(1):309-320. DOI: 10.2147/COPD.S76293

36. Campo G, Pavasini R, Malagù M, Mascetti S, Biscaglia S, Ceconi C, et al. Chronic Obstructive Pulmonary Disease and Ischemic Heart Disease Comorbidity: Overview of Mechanisms and Clinical Management. Cardiovasc Drugs Ther. 2015 Apr;29(2):147-57. DOI: 10.1007/s10557-014-6569-y

37. Rydén L, Roos A, Holzmann MJ. Chronic Myocardial Injury and Risk for Stroke. Am J Med. 2019 Jul;132(7):833-9. DOI: 10.1016/j.amjmed.2019.01.027

38. Korosoglou G, Lehrke S, Mueller D, Hosch W, Kauczor HU, Humpert PM, et al. Determinants of troponin release in patients with stable coronary artery disease: Insights from CT angiography characteristics of atherosclerotic plaque. Heart. 2011 May;97(10):823-31. DOI: 10.1136/hrt.2010.193201 